\title{
Scaling behavior of the momentum distribution of a quantum Coulomb system in a confining potential
}

\author{
J. A. E. Bonart $\odot,{ }^{1,2}$ W. H. Appelt, ${ }^{1}$ D. Vollhardt, ${ }^{1}$ and L. Chioncel $\odot^{1,3}$ \\ ${ }^{1}$ Theoretical Physics III, Center for Electronic Correlations and Magnetism, Institute of Physics, \\ University of Augsburg, 86135 Augsburg, Germany \\ ${ }^{2}$ BMW AG, 80807 Munich, Germany \\ ${ }^{3}$ Augsburg Center for Innovative Technologies, University of Augsburg, 86135 Augsburg, Germany
}

(Received 12 May 2020; accepted 1 July 2020; published 13 July 2020)

\begin{abstract}
We calculate the single-particle momentum distribution of a quantum many-particle system in the presence of the Coulomb interaction and a confining potential. The region of intermediate momenta, where the confining potential dominates, marks a crossover from a Gaussian distribution valid at low momenta to a power-law behavior valid at high momenta. We show that for all momenta the momentum distribution can be parametrized by a $q$-Gaussian distribution whose parameters are specified by the confining potential. The real-space paircorrelation function calculated in this way can, in principle, be used to construct improved exchange-correlation functionals to solve electronic structure problems. Furthermore, we find that the functional form of the probability of transitions between the confined ground state and the $n$th excited state is invariant under scaling of the ratio $Q^{2} / v_{n}$, where $Q$ is the transferred momentum and $v_{n}$ is the corresponding excitation energy. Using the scaling variable $Q^{2} / v_{n}$ the maxima of the transition probabilities can also be expressed in terms of a $q$-Gaussian.
\end{abstract}

DOI: 10.1103/PhysRevB.102.024306

\section{INTRODUCTION}

The single-particle momentum distribution plays an important role in our understanding of the ground-state properties of quantum many-particle systems [1,2]. It is defined as the average number of particles with momentum $\mathbf{k}$ in an $N$-particle system, $n(\mathbf{k})=\left\langle\Psi\left|\sum_{\sigma} a_{\mathbf{k} \sigma}^{\dagger} a_{\mathbf{k} \sigma}\right| \Psi\right\rangle$. Here the normalized $N$-particle state of the system is represented by $|\Psi\rangle$ and $a_{\mathbf{k} \sigma}^{\dagger}\left(a_{\mathbf{k} \sigma}\right)$ are the creation (annihilation) operators for particles with momentum $\mathbf{k}$ and spin projection $\sigma$. In real space the one-particle density matrix

$$
\rho\left(\mathbf{x}_{1}, \mathbf{x}_{1}^{\prime}\right)=\int \prod_{i=2}^{N} d \mathbf{x}_{i} \psi^{\dagger}\left(\mathbf{x}_{1}, \mathbf{x}_{2}, \ldots, \mathbf{x}_{N}\right) \psi\left(\mathbf{x}_{1}^{\prime}, \mathbf{x}_{2}, \ldots, \mathbf{x}_{N}\right)
$$

measures the change of the $N$-particle wave function when a particle is moved from $\mathbf{x}_{1}^{\prime}$ to $\mathbf{x}_{1}$ while all other particles are fixed. In homogeneous systems this two-point function depends only upon the separation $\rho\left(\mathbf{x}_{1}, \mathbf{x}_{1}^{\prime}\right)=\rho\left(\left|\mathbf{x}_{1}-\mathbf{x}_{1}^{\prime}\right|\right)$. Accordingly, the momentum distribution and the one-particle density matrix are related by Fourier transformation

$$
n(\mathbf{k})=\int d \mathbf{x}_{1} \int d \mathbf{x}_{1}^{\prime} e^{i \mathbf{k} \cdot\left(\mathbf{x}_{1}-\mathbf{x}_{1}^{\prime}\right)} \rho\left(\mathbf{x}_{1}-\mathbf{x}_{1}^{\prime}\right) .
$$

The momentum distribution, Eq. (2), is determined by a product of two field operators whose short-distance behavior can be calculated exactly using renormalization group methods [3-6]. The later techniques, when applied in nuclear physics, decouple the low- from the high-momentum degrees of freedom and leave the scattering cross section invariant $[2,7-11]$. Furthermore, the nuclear momen- tum distributions calculated within the impulse approximation $[12,13]$ provide universal scaling laws for the highmomentum tails [2] of one- and two-particle momentum distributions $[4,14]$. These tails are the consequence of shortrange correlations in the nuclear wave functions [15]. Renormalization group arguments [16,17] have also shown that high-momentum tails of momentum distributions factorize into a product between a universal function of momentum, which is determined by two-particle physics and a factor depending on the low-momentum structure of the many-body state [6]. This observation goes back to Kimball $[18,19]$ who pointed out that when two particles are sufficiently close their interaction dominates, and the two-particle Schrödinger equation provides a reasonable starting point to compute quantum mechanical observables from the knowledge of the pair wave function.

Experimental measurements of $n(\mathbf{k})$ involve inelastic scattering processes with energy and momentum transfers larger than the characteristic lengthscale of the scatterer. They determine the double differential scattering cross section $d^{2} \sigma / d \Omega d \omega$ for a given infinitesimal solid angle $d \Omega$ and energy $d \omega$ of the scattered particle, respectively. The incident energy and the scattering angle are fixed during the experiment, and the scattering cross section is measured as a function of the transferred momentum and energy. The data analysis of the measured scattering cross section generally employs the impulse approximation [12,13], which assumes that a single particle is struck by the scattering probe, and that the particle recoils freely from the collision. Within the impulse approximation the scattering cross section is proportional to the Compton profile $d^{2} \sigma / d \Omega d \omega \propto J\left(k_{z}\right)$. The latter 
quantity can be calculated directly by integrating the momentum distribution $n(\mathbf{k})$ in a plane perpendicular to the scattering vector $k_{z}: J\left(k_{z}\right)=\iint n(\mathbf{k}) d k_{x} d k_{y}$. The proportionality implies that, whenever the measured scattering cross section is modelled within the impulse approximation [1,2] and is found to be invariant under some scaling transformation, the Compton profile will show the same scaling behavior.

In this paper we investigate whether the momentum distribution of a Coulomb system, which yields the Compton profile by integration, also shows scaling behavior. In high-energy physics it is well known that the scaling of the scattering cross section is a consequence of confinement ("Bjorken scaling"). Indeed, by assuming the existence of a simple confining potential for two point particles, Elitzur and Susskind [20] derived the scaling behavior of the resonance excitations found experimentally in deep inelastic reactions [8,21]. Making use of Kimball's observation [18,19] we will therefore compute the momentum distribution of two interacting electrons by numerically solving the two-particle Schrödinger equation for a repulsive Coulomb interaction in the presence of a confining potential. In the following we work in atomic units, where the unit length is $a_{0}=$ $1 \operatorname{Bohr}\left(0.529167 \times 10^{-10} \mathrm{~m}\right)$, the unit of mass is the electron mass $m$, and the unit of energy is 1 Hartree $(1 \mathrm{Ha}=$ $27.2113 \mathrm{eV})$. To keep the investigation general we consider an algebraic confining potential of the model form $V(x)=$ $\alpha\left|x / a_{0}\right|^{\eta}$. The condition $\eta>0$ ensures that the potential produces bound states, and we chose $\alpha=1 \mathrm{Ha}$. We will show that the momentum distribution of a quantum many-particle system interacting via the Coulomb interaction in the presence of a confining potential can be parametrized by a $q$-Gaussian distribution whose parameters are determined by the confining potential.

In Sec. II we compute the high-momentum tails of the momentum distribution, Eq. (2), in the ground state and show that they obey scaling relations. A crossover in the momentum density from an ordinary Gaussian distribution at small momenta to a power-law behavior at larger momenta occurs when the Coulomb potential dominates the confinement. In the cross-over region of size $\approx 5 / a_{0}$ we find that the shape of the momentum distribution is described by a $q$ Gaussian with $k$-dependent parameters. At large momenta we recover the exact results obtained by renormalization group methods $[4-6,16,17]$. Using the solutions of the two-particle Schrödinger equation of Sec. II we show in Sec. III that when the confinement dominates the Coulomb interaction, the transition matrix elements into excited states (nth-bound level) due to momentum absorption also obey $q$-Gaussian distributions, and we connect the $q$-parameter to the shape of the confining potential. The $q$-Gaussian used to parametrize the momentum distribution is characterized by parameters which are different from those used to fit the transition probabilities between the bound states. Finally, in Sec. IV we relate these results to the recent observation [22] that the Compton profiles of all alkali elements can be collapsed onto a single curve which is described by a $q$-Gaussian. Furthermore, we point out that the pair-correlation function calculated in our approach in real space can be used to construct improved exchange-correlation functionals to solve electronic structure problems.

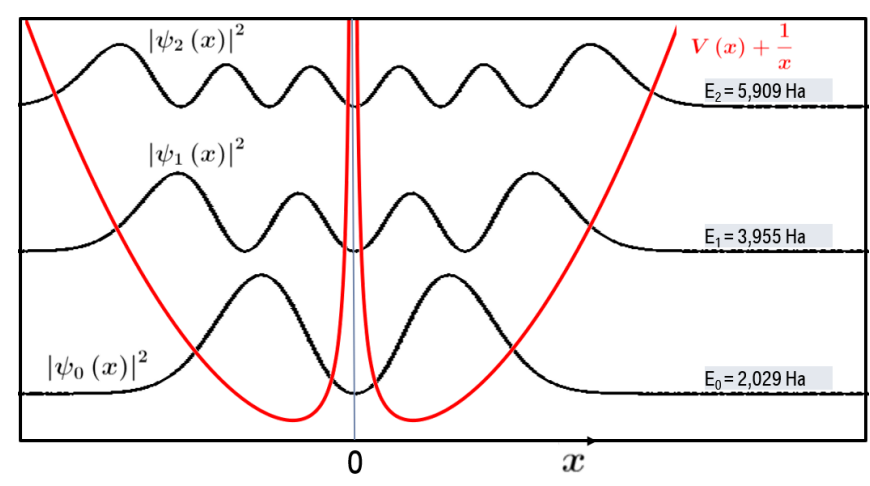

FIG. 1. Probability density $\left|\psi_{n}(x)\right|^{2}$ of the pair wave functions for $n=0,1,2$ obtained from the solutions of Eq. (4). Red solid line: Confining potential $V(x)$, with $\eta=2$, together with the repulsive Coulomb term $1 / x$, which is singular at $x=0$. The corresponding energies $E_{n}$ of the ground state and the first excited states are shown on the right. For a better visibility the probability densities are separated along the vertical axis.

\section{GROUND STATE: KIMBALL'S APPROACH TO THE MOMENTUM DISTRIBUTION}

We consider nonrelativistic electrons with Coulomb interaction whose Hamiltonian reads $[18,19]$

$$
H=-\frac{\hbar^{2}}{2 m} \sum_{i=1}^{N} \nabla_{i}^{2}-\sum_{i=1}^{N} \sum_{I=1}^{N_{I}} \frac{e^{2} Z_{I}}{\left|\mathbf{x}_{i}-\mathbf{R}_{I}\right|}+\sum_{i<j} \frac{e^{2}}{\left|\mathbf{x}_{i}-\mathbf{x}_{j}\right|} .
$$

Here $\mathbf{x}_{i}$ and $\mathbf{R}_{I}$ are the electronic and nuclear coordinates, respectively, $e Z_{I}$ is the charge of the $I$ th nucleus, and $m$ is the mass of an electron. The first term is the kinetic energy of the electrons, and the remaining two terms represent the Coulomb repulsion between the electrons and nuclei, and between the electrons themselves. The eigenstates of the Hamiltonian (3) are time-independent wave functions $\psi\left(\mathbf{x}_{1}, \mathbf{x}_{2}, \ldots, \mathbf{x}_{N}\right)$ which are normalized to the volume of the system. Here periodic boundary conditions are assumed and spin indices are suppressed. Following the idea of Kimball $[18,19]$, the behavior of the wave function at large momenta is determined by the dependence at small distances between two particles. As the distance approaches zero, the dynamics of adjacent particles is dominated by the Coulomb force.

Instead of the explicit electron-nucleon attraction we consider an effective confining potential $V(r)$ whose nature or origin we do not further specify since we merely wish to understand the consequences of the confining potentials, e.g., possible scaling properties. Introducing relative coordinates $\mathbf{x}=\mathbf{x}_{1}-\mathbf{x}_{2}$ and center-of-mass coordinates $\mathbf{X}=\left(\mathbf{x}_{1}+\mathbf{x}_{2}\right) / 2$ for the two particles as well as the reduced mass $\mu=1 / 2$, the Schrödinger equation becomes

$$
\left(-\frac{\partial^{2}}{\partial x^{2}}+V(x)+\frac{1}{x}\right) \psi_{n}(x)=E_{n} \psi_{n}(x) .
$$

The solutions to Eq. (4) are denoted by $\psi_{n}(x)$ with the corresponding eigenenergy $E_{n}$, are shown in Fig. 1. The ansatz for the total wave function introduced by Kimball $[18,19]$ separates the dependence on the relative coordinates from that 
of the center-of-mass motion

$$
\begin{aligned}
\Psi_{n} & \equiv \Psi_{n}\left(\mathbf{x}_{1}, \mathbf{x}_{2}, \mathbf{x}_{3}, \ldots, \mathbf{x}_{N}\right) \\
& =\Psi_{n}\left(\mathbf{x}, \mathbf{X}, \mathbf{x}_{3}, \ldots, \mathbf{x}_{N}\right) \\
& \simeq \Phi\left(\mathbf{X}, \mathbf{x}_{3}, \ldots, \mathbf{x}_{N}\right) \psi_{n}(\mathbf{x}) .
\end{aligned}
$$

The one-particle density matrix for the relative coordinates is then given by

$$
\begin{aligned}
\rho\left(\mathbf{x}, \mathbf{x}^{\prime}\right) \simeq & \int d \mathbf{X} \prod_{i=3}^{N} d \mathbf{x}_{i} \Phi^{\dagger}\left(\mathbf{X}, \mathbf{x}_{3}, \ldots, \mathbf{x}_{N}\right) \psi_{n}^{\dagger}(\mathbf{x}) \\
& \Phi\left(\mathbf{X}, \mathbf{x}_{3}, \ldots, \mathbf{x}_{N}\right) \psi_{n}\left(\mathbf{x}^{\prime}\right) \\
= & \bar{\rho} \psi_{n}^{\dagger}(\mathbf{x}) \psi_{n}\left(\mathbf{x}^{\prime}\right),
\end{aligned}
$$

where $\bar{\rho}$ represents the integral of the $N$-particle wave function over all coordinates $\mathbf{x}_{i}$ and $\mathbf{X}$.

From $\rho\left(\mathbf{x}, \mathbf{x}^{\prime}\right)$ we obtain the two-particle ("pair") correlation function $g(\mathbf{x})$, which is defined as $g(\mathbf{x})=\rho(\mathbf{x}, \mathbf{x})=$ $\bar{\rho}\left|\psi_{n}(\mathbf{x})\right|^{2}$ and is proportional to the probability of two particles being at a distance $\mathbf{x}$ (see Fig. 1). The momentum distribution is computed according to Eq. (2).

Figure 2(a) shows the momentum dependence of $n(k)$ for the confining potential $V(x)=\alpha\left|x / a_{0}\right|^{\eta}$ with $\eta=2$, where $k$ is the magnitude of $\mathbf{k}$. One clearly sees a Gaussian regime at small $k$, which is followed by a crossover into the asymptotic region at large $k$. To specify the momentum dependence by a unique functional form we fit the momentum distribution with a $q$-Gaussian [23]. To determine the $(q, \beta)$ parameters we collect $k$ values into "bins" that are characterized by an average value $\bar{k}$. The later quantity is computed from the interval in which the fitting to the $q$-Gaussian form is performed and which contains at least five points in the low momentum and an order of magnitude more (50) points in the asymptotic region. The initial fitting parameters of the $i$ th bin are the final parameters of the $(i-1)$ th $\bar{k}$-bin. For a given bin the same values $q(\bar{k}), \beta(\bar{k})$ parametrize the momentum dependence as [24]

$$
n_{q(\bar{k}), \beta(\bar{k})}(k)=\frac{1}{\sqrt{2} \beta(\bar{k}) C_{q(\bar{k})}} \exp _{q(\bar{k})}\left[-\beta(\bar{k}) k^{2}\right]
$$

For arbitrary values of $q$, the $q$-exponential is defined as $\exp _{q}(x)=[1+(1-q) x]^{1 /(1-q)}$. In Eq. (7) $C_{q(\bar{k})}$ is a normalization constant and $\beta(\bar{k})$ controls the width of the distribution

In Fig. 2(b) we plot the momentum dependence of the $(q, \beta)$-parameters. We see that for low momenta $q(\bar{k})=1$ while $\beta(\bar{k})=0.49$. In fact, the $\exp _{q}$-function becomes the exponential function in the limit of $q \rightarrow 1$, whereby the Gaussian distribution is recovered. The low momentum region corresponds to large distances. In this case the Coulomb interaction is negligible and the solutions become plane waves $[18,19]$, which form Gaussian wave packages leading to a Gaussian momentum dependence. In the crossover region, i.e., in the range $\bar{k}_{\text {min }} \approx 1.0 / a_{0}$ to $\bar{k}_{\max } \approx 5.0 / a_{0}$, a smooth transition between Gaussian and power-law behavior is observed in the momentum dependence of the $(q, \beta)$ parameters.

For large values of $k>k_{\max }$ the $q$-Gaussian distribution has a power-law dependence $\left.f_{q, \beta}(x)\right|_{x \rightarrow \infty} \propto x^{2 /(1-q)}$, which in our case amounts to constant values of the parameters $q(\bar{k})=$ 1.50 and $\beta(\bar{k})=0.06$. Thus, at large momenta the power-law
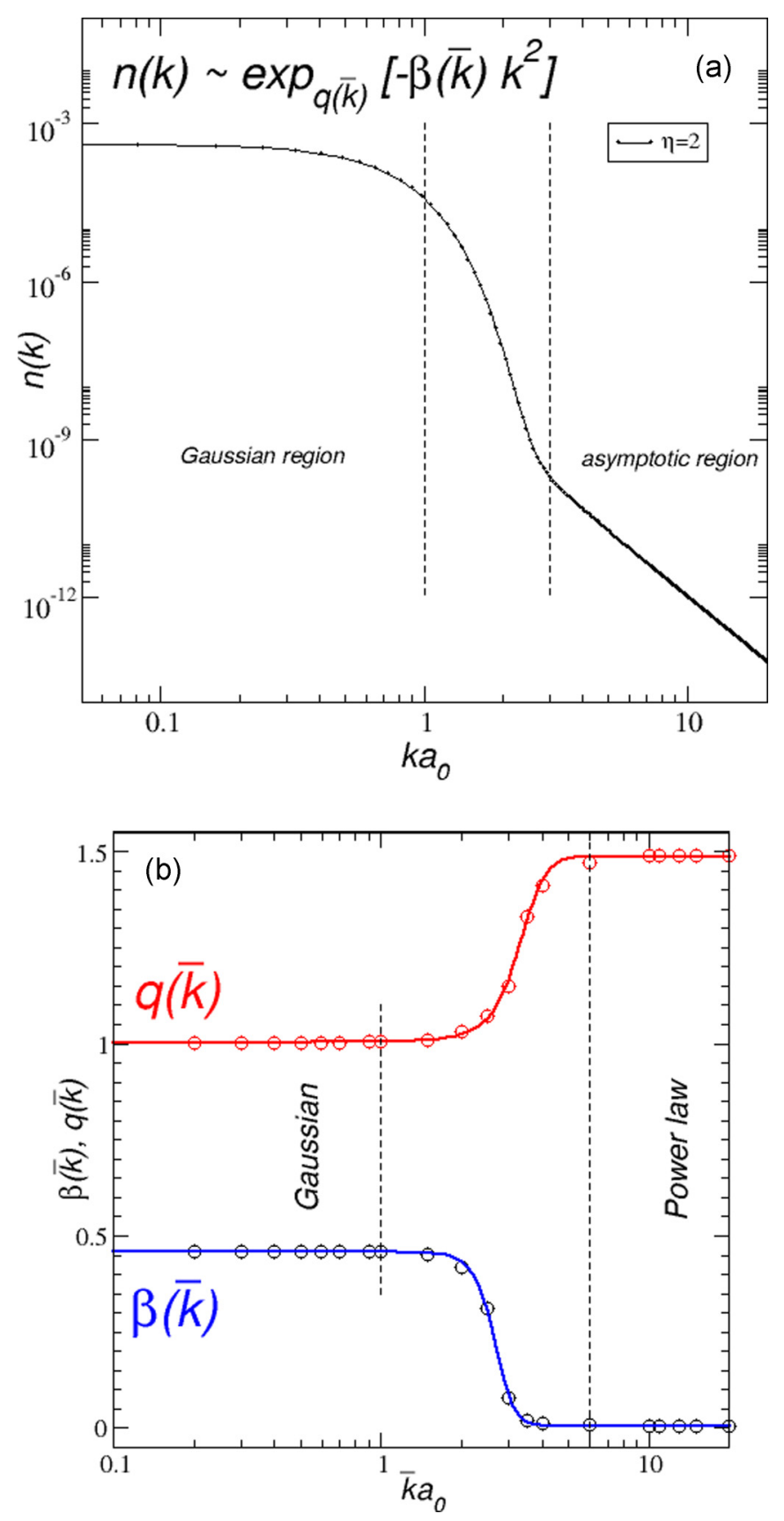

FIG. 2. (a) Computed momentum distribution $n(k)$ of electrons in the presence of a confining potential $(\alpha=1 \mathrm{Ha}, \eta=2)$. The momentum distribution is fitted by a $q$-Gaussian in the entire momentum region. (b) Dependence of the $q, \beta$-parameters on the average momentum $\bar{k}$. The low momentum region shows an ordinary Gaussian behavior $(q=1)$. In the intermediate region $\left(k_{\min } \approx 1.0 / a_{0}<k<\right.$ $\left.k_{\text {max }} \approx 5.0 / a_{0}\right) q$-Gaussian type of fits are possible with momentumdependent parameters. At large momenta the power-law dependence is recovered.

behavior is recovered $[18,19]$, and the asymptotic behavior of the momentum density agrees with the result obtained by the renormalization group approach $[16,17]$. We note that the $q$-Gaussian fits and the corresponding $q(\bar{k}), \beta(\bar{k})$-parameters differ for different values of the confining potentials (values of the parameter $\eta)$. Nonetheless, constant values of $q(\bar{k}), \beta(\bar{k})$ 
are obtained for the low momentum region as well as the asymptotic region.

In the following section we will show that the transition matrix elements between the ground state and the $n$ th-energy level caused by absorption of a momentum, calculated from the solutions of Eq. (4), also obey scaling. This result was previously found by Elitzur and Susskind within a simplified parton model [20]. Here we compute the full transition probability and demonstrate that the scaling functions for the maxima of the transition probabilities can also be expressed by $q$-Gaussians. Since transition probabilities are connected to the scattering cross section from which the Compton profiles follow, our calculation proves that the Compton profile also scales, provided that the potential energy due to the confinement dominates the Coulomb interaction.

\section{EXCITED STATES: ELITZUR-SUSSKIND BOUND-STATE RESONANCES}

Elitzur and Susskind employed a simple confining potential [20] to explain the scaling of the resonance excitations in deep inelastic reactions [8,21]. In their simplified model the probability for transitions between the bound states in the confining potential was computed using the dipole approximation and was shown to be compatible with the scaling of resonant excitations $[20,25,26]$ in deep inelastic reactions. Contrary to Ref. [20] in which the Wentzel-Kramers-Brillouin (WKB) approximation was used, we solve Eq. (4) numerically for a pair of point particles with masses $m_{1}, m_{2}$ in a potential which is sufficiently deep to bind states. We assume that the momentum $Q$ is absorbed by one of the particles, and the bound pair is lifted to the $n$ th-level, at an energy $v_{n}=$ $E_{n}-E_{0}$. Using the solutions of Eq. (4) we evaluate the matrix elements $F\left(v_{n}, Q^{2}\right)$ for the transition into the $n$th bound level due to the absorption of a momentum $\mathbf{Q}$. We note that the solutions of Eq. (4) produce bound states [27] irrespective of the sign of the confining potential. The transition probability is the square of the transition matrix element

$$
T\left(v_{n}, Q^{2}\right)=\left|\left\langle\psi_{n}\left|e^{i \mathbf{Q} \cdot \mathbf{x}_{1}}\right| \psi_{0}\right\rangle\right|^{2}=\left|\left\langle\psi_{n}\left|e^{i \mathbf{Q}_{1} \mathbf{x}}\right| \psi_{0}\right\rangle\right|^{2} .
$$

Here $\psi_{0}$ and $\psi_{n}$ describe the ground state and the $n$th bound state of the confining potential, respectively, with $\mathbf{Q}_{i}=$ $\mathbf{Q} m_{i} /\left(\sum_{i} m_{i}\right)$. For electrons $m_{1}=m_{2}$ whereby $\mathbf{Q}_{i}=\mathbf{Q} / 2$ as the scaled momentum. For finite $Q^{2}$ the transition probability $T\left(v_{n}, Q^{2}\right)$ leads to $n$ discrete resonances. The numerical results are presented in Fig. 3.

In Ref. [20] it was noted that the matching between the phase of $\psi_{n}$ and the exponential factor $e^{i \mathbf{Q}_{1} \mathbf{x}}$ implies a linear relation ("scaling direction") between the square of the transferred momentum $Q^{2}$ and the excitation energy $v_{n}$. The scaling direction therefore represents a line in the $\left(v_{n}, Q^{2}\right)$ plane which we illustrate in the upper part of Fig. 3. For any other direction in the $\left(v_{n}, Q^{2}\right)$-plane the transition probability decays exponentially (no phase matching). This result was already derived in Ref. [20] within the WKB approximation, where the line has slope 1 . It is interesting to note that this result is not exactly reproduced in the present calculations, where the Coulomb interaction is taken into account.

From the fact that the scaling direction is essentially a line in the $\left(v_{n}, Q^{2}\right)$-plane along which the transition probability is

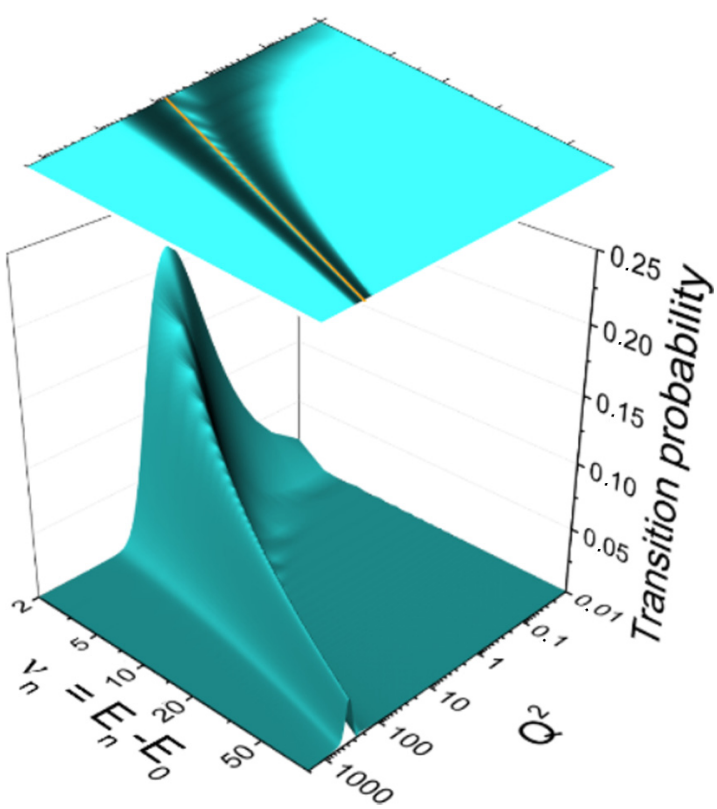

FIG. 3. Transition probability $T\left(v_{n}, Q^{2}\right)$ computed for the confining potential with $\eta=2$ in the presence of the Coulomb interaction. The scaling direction is seen by projection into the $\left(v_{n}, Q^{2}\right)$ plane.

maximal, we can identify the ratio $Q^{2} / v_{n} \equiv \gamma$ as a scaling variable. We fitted the maxima along the scaling direction using a $q$-Gaussian form

$$
T\left(v_{n}, Q^{2}\right):=T_{q, \beta}\left(Q^{2} / v_{n}\right)=\frac{1}{\sqrt{2} \beta C_{q}} \exp _{q}\left(-\beta Q^{2} / v_{n}\right) .
$$

In the limit of large momenta we find

$$
\left.\lim _{Q^{2} \rightarrow \infty} T_{q, \beta}\left(Q^{2} / v_{n}\right)\right|_{v_{n}=Q^{2} / \gamma} \propto \gamma^{2 /(1-q)},
$$

i.e., the $q$-Gaussian takes the form of a power law, which is characterized by scale invariance. In Fig. 4(a) we show the $q$-Gaussian fits to the maxima of the transition probability for different confining potentials, i.e., different values of $\eta$. In the limit of large momentum transfer $Q^{2} \rightarrow \infty$ we obtain, for all $\eta$ values, power laws along the "scaling direction" with a particular scaling exponent. Due to the scaling property they are all equivalent up to constant factors. This behavior is presented in Fig. 4(b) where the $q$-logarithm of the transition probability is plotted against the scaling variable $Q^{2} / v_{n}$. Here $\ln _{q}$ is the $q$-analog of the logarithm defined by $\ln _{q} x:=\left(x^{1-q}-1\right) /(1-q)$. This plot is seen to produce an essentially linear relation between the scaled transition probabilities, which collapse onto a single curve. Deviations are due to finite-size effects and numerical precision in the high $Q$-regime.

The $q$ and $\beta$ values for the fits in Fig. 4 are shown in Figs. 5(a) and 5(b). We see that $q$ and $\beta$ increase linearly for growing $\eta$ values. With increasing $\eta$ the confining potential becomes steeper as the interparticle distance increases. Therefore one expects that the wave function (eigenfunction of $H$ ) is more localized, which leads to a slower decay of the transition probabilities at high momenta. 

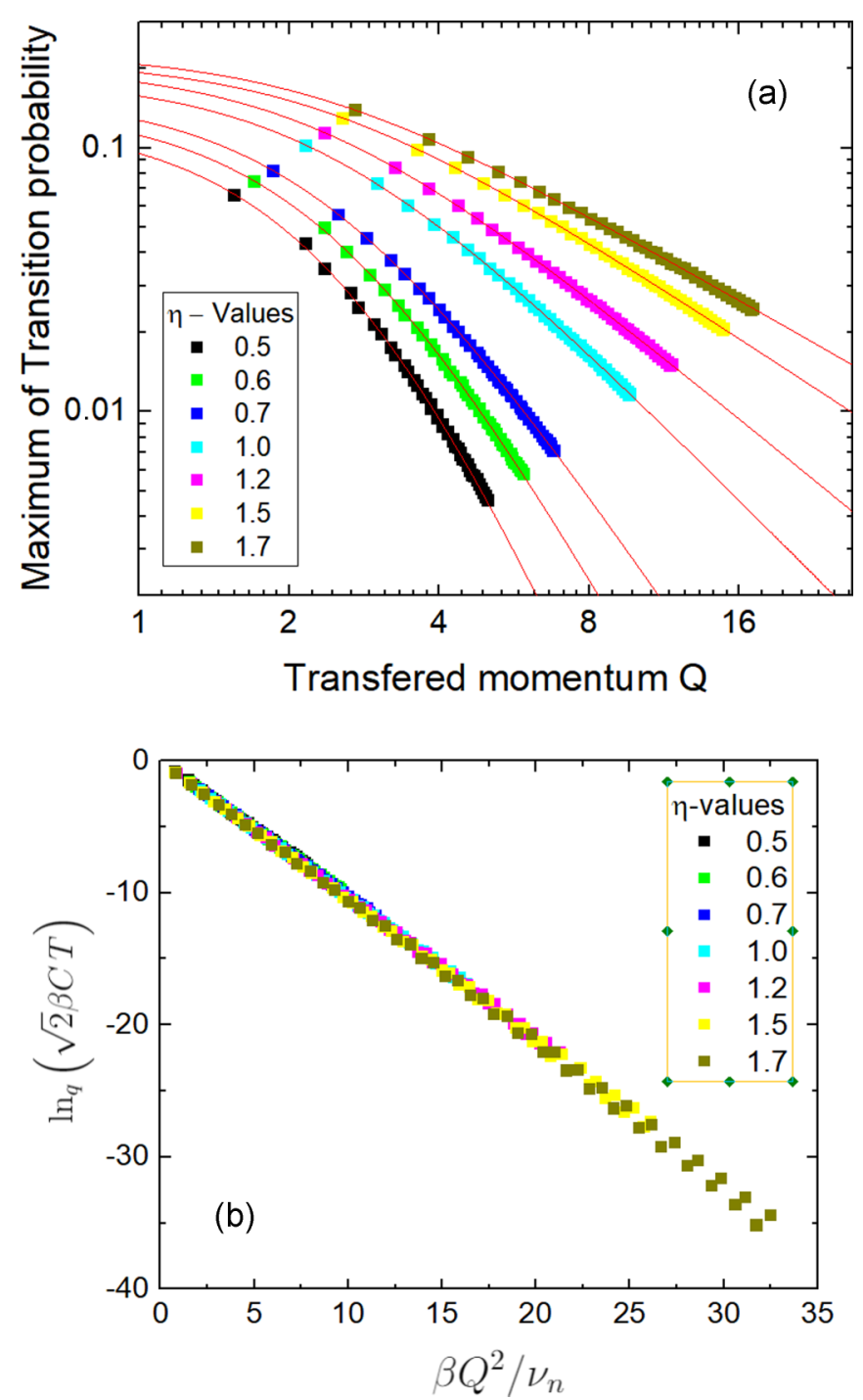

FIG. 4. (a) The maxima of the transition probabilities corresponding to each transferred momentum $Q$ are plotted for different confining potential strengths $\eta$. Dots are numerical data, while the red lines show the fit with a $q$-Gaussian distribution. (b) By scaling all maxima the transition probabilities collapse onto a single curve.

\section{DISCUSSION}

The results presented in this paper were initiated by the question whether, and under what conditions, the momentum distribution of a Coulomb systems shows scaling behavior. Following Kimball's approach $[18,19]$, we computed the momentum distribution of two interacting electrons by numerically solving the two-particle Schrödinger equation for a repulsive Coulomb interaction in the presence of a confining potential. We found that $n(\mathbf{k})$ can be parametrized by a $q$-Gaussian in the entire momentum range. This crossover region connects the low-momenta region, described by an ordinary Gaussian momentum dependence, with a power-law behavior at large momenta. In the confinement dominated (intermediate) momentum region we used the method of Elitzur and Susskind [20] and demonstrated that bound-state
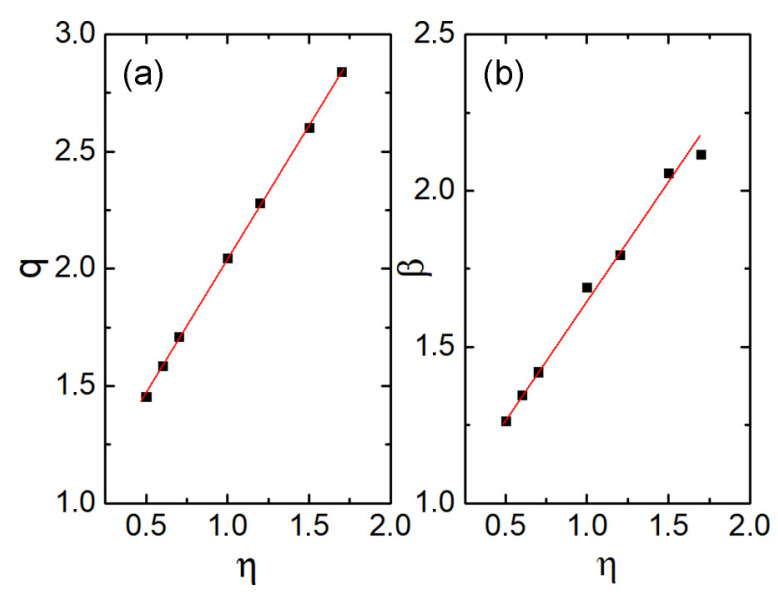

FIG. 5. (a), (b) The dependence of $q / \beta$ for different $\eta$ values. The red line is a guide to the eye.

resonances also show scaling behavior. In particular, we demonstrated that $q$-Gaussians are suitable scaling functions for the maxima of the transition probability. Indeed, the $q$ Gaussian behavior is expected to enter in this investigation since it is the natural mathematical function that can describe fat-tail distributions, whose asymptotic momentum dependence is not exponential but is described, for example, by a power law. Whenever the Coulomb interaction dominates the confining potential (in the large-momenta region) our results recover the exact analytic results obtained by renormalization group techniques $[6,16,17]$. It would be desirable to gain insight into the numerically derived scaling properties also within an analytic approach.

Using density functional theory (DFT) [28-31] in combination with the impulse approximation we recently showed that Compton profiles of the first column elements of the periodic table can be collapsed onto a single curve [22] which can be fitted by a $q$-Gaussian with element specific $(q, \beta)$ parameters. In that study we did not address the questions of why there should be scaling behavior at all, and why the $q$-Gaussian was found to be a suitable scaling function. In view of the fact that in the electronic band theory of solids the periodic ionic potential provides a natural confining potential, the results of the present paper may provide an explanation of the unexpected scaling behavior of the Compton profiles of the alkali elements [22].

For the application of the DFT [28-31] in the framework of the Kohn-Sham ansatz the knowledge of the exchangecorrelation functional is crucial. A central quantity is the socalled coupling constant integrated pair-correlation function. It accounts for the electronic correlations contribution into the kinetic energy [30] and is the input to the derivation of the gradient corrected functionals [32,33]. The contribution of electronic correlations to the kinetic energy has been analyzed also in momentum space [34], and the limits of large and small momenta were discussed and found to be in agreement with results of Kimball [18,19]. In fact, according to our investigation which extends the work of Kimball by including confining potentials, a $q$-Gaussian fit for the momentum density is possible for every value of the momentum, and the 
somewhat arbitrary separation between short- and long-range decomposition can be avoided. We expect that the concept of scaling of the momentum distribution in terms of a $q$-Gaussian can be further developed such that it actually provides new exchange-correlation functionals.

\section{ACKNOWLEDGMENTS}

Financial support by the Deutsche Forschungsgemeinschaft through TRR80 (project F6) Project No. 107745057 and useful discussions with M. Sekania and M. Kollar are gratefully acknowledged.
[1] R. N. Silver and P. E. Sokol, Momentum Distributions (Springer, New York, 1989).

[2] G. B. West, Phys. Rep. 18, 263 (1975).

[3] K. G. Wilson and W. Zimmermann, Commun. Math. Phys. 24, 87 (1972).

[4] M. Alvioli, C. Ciofi degli Atti, L. P. Kaptari, C. B. Mezzetti, H. Morita, and S. Scopetta, Phys. Rev. C 85, 021001(R) (2012).

[5] M. Vanhalst, J. Ryckebusch, and W. Cosyn, Phys. Rev. C 86, 044619 (2012).

[6] E. R. Anderson, S. K. Bogner, R. J. Furnstahl, and R. J. Perry, Phys. Rev. C 82, 054001 (2010).

[7] G. Reiter and R. Silver, Phys. Rev. Lett. 54, 1047 (1985).

[8] J. D. Bjorken, Phys. Rev. 179, 1547 (1969).

[9] J. D. Bjorken, Phys. Rev. 163, 1767 (1967).

[10] H. D. Politzer, Phys. Rev. Lett. 30, 1346 (1973).

[11] H. D. Politzer, Phys. Rep. 14, 129 (1974).

[12] G. F. Chew and G. C. Wick, Phys. Rev. 85, 636 (1952).

[13] J. Ashkin and G. C. Wick, Phys. Rev. 85, 686 (1952).

[14] M. Vanhalst, W. Cosyn, and J. Ryckebusch, Phys. Rev. C 84, 031302(R) (2011).

[15] S. C. Pieper, R. B. Wiringa, and V. R. Pandharipande, Phys. Rev. C 46, 1741 (1992).

[16] S. K. Bogner and D. Roscher, Phys. Rev. C 86, 064304 (2012).

[17] J. Hofmann, M. Barth, and W. Zwerger, Phys. Rev. B 87, 235125 (2013).

[18] J. C. Kimball, Phys. Rev. A 7, 1648 (1973).

[19] J. C. Kimball, J. Phys. A: Math. Gen. 8, 1513 (1975).

[20] M. Elitzur and L. Susskind, Phys. Rev. D 6, 2003 (1972).

[21] E. D. Bloom and F. J. Gilman, Phys. Rev. Lett. 25, 1140 (1970).
[22] M. Sekania, W. H. Appelt, D. Benea, H. Ebert, D. Vollhardt, and L. Chioncel, Physica A: Statistical Mechanics Applications 489, 18 (2018).

[23] C. Tsallis, J. Stat. Phys. 52, 479 (1988).

[24] In this context we note that the ground-state wave function of a quantum particle in a Coulomb potential has the form of a $q$-Gaussian in momentum space [35]. Furthermore the distribution of the energies of all elements of the periodic table were also observed to follow a $q$-Gaussian [36].

[25] M. Elitzur, Phys. Rev. D 3, 2166 (1971).

[26] J. Kogut, Phys. Rev. D 8, 3029 (1973).

[27] S. M. Mahajan and A. Thyagaraja, J. Phys. A: Math. Gen. 39, L667 (2006).

[28] P. Hohenberg and W. Kohn, Phys. Rev. 136, B864 (1964).

[29] W. Kohn, Rev. Mod. Phys. 71, 1253 (1999).

[30] R. O. Jones and O. Gunnarsson, Rev. Mod. Phys. 61, 689 (1989).

[31] R. O. Jones, Rev. Mod. Phys. 87, 897 (2015).

[32] J. P. Perdew, K. Burke, and M. Ernzerhof, Phys. Rev. Lett. 77, 3865 (1996).

[33] J. P. Perdew, K. Burke, and Y. Wang, Phys. Rev. B 54, 16533 (1996).

[34] P. Gori-Giorgi and J. P. Perdew, Phys. Rev. B 66, 165118 (2002).

[35] C. Vignat, A. Plastino, A. R. Plastino, and J. S. Dehesad, Physica A: Statistical Mechanics Applications 391, 1068 (2012).

[36] C. H. S. Amadora and L. S. Zambranob, Physica A: Statistical Mechanics Applications 389, 3866 (2010). 